\title{
THREE RESULTS ON THE REGULARITY OF THE GURVES THAT ARE INVARIANT BY AN EXAGT SYMPLECTIC TWIST MAP
}

\author{
by M.-C. ARNAUD
}

\begin{abstract}
A theorem due to G. D. Birkhoff states that every essential curve which is invariant under a symplectic twist map of the annulus is the graph of a Lipschitz map. We prove: if the graph of a Lipschitz map $h: \mathbf{T} \rightarrow \mathbf{R}$ is invariant under a symplectic twist map, then $h$ is a little bit more regular than simply Lipschitz (Theorem 1); we deduce that there exists a Lipschitz map $h: \mathbf{T} \rightarrow \mathbf{R}$ whose graph is invariant under no symplectic twist map (Corollary 2).

Assuming that the dynamic of a twist map restricted to a Lipschitz graph is bi-Lipschitz conjugate to a rotation, we obtain that the graph is even $\mathrm{C}^{1}$ (Theorem 3 ).

Then we consider the case of the $\mathrm{C}^{0}$ integrable symplectic twist maps and we prove that for such a map, there exists a dense $\mathrm{G}_{\delta}$ subset of the set of its invariant curves such that every curve of this $\mathrm{G}_{\delta}$ subset is $\mathrm{C}^{1}$ (Theorem 4 ).
\end{abstract}

\section{CONTENTS}

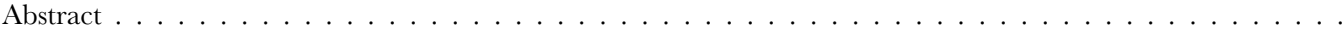

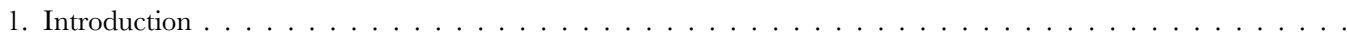

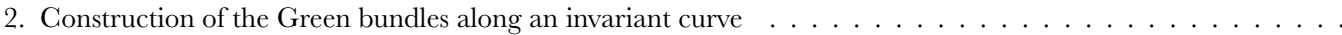

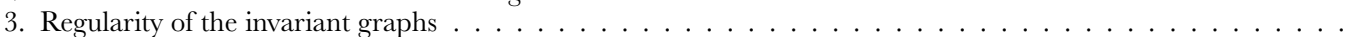

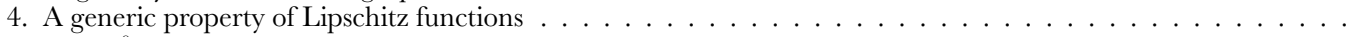

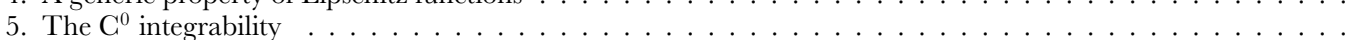

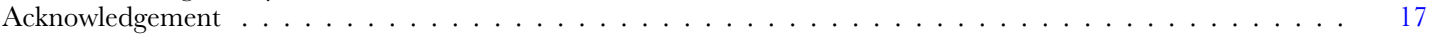

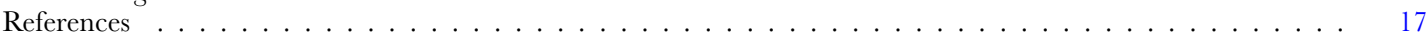

\section{Introduction}

The exact symplectic twist maps were studied for a long time because they represent (via a symplectic change of coordinates) the dynamic of the generic symplectic diffeomorphisms of surfaces near their elliptic periodic points (see [3]).

Let us introduce some notations and definition:

Notations.

- $\mathbf{T}=\mathbf{R} / \mathbf{Z}$ is the circle.

- $\mathbf{A}=\mathbf{T} \times \mathbf{R}$ is the annulus and an element of $\mathbf{A}$ is denoted by $(\theta, r)$.

- $\mathbf{A}$ is endowed with its usual symplectic form, $\omega=d \theta \wedge d r$.

- $\pi: \mathbf{T} \times \mathbf{R} \rightarrow \mathbf{T}$ is the projection and $\tilde{\pi}: \mathbf{R}^{2} \rightarrow \mathbf{R}$ its lift.

Definition. - $A \mathrm{C}^{1}$ diffeomorphism $f: \mathbf{A} \rightarrow \mathbf{A}$ of the annulus which is isotopic to identity is a positive twist map if, for any given lift $\tilde{f}: \mathbf{R}^{2} \rightarrow \mathbf{R}^{2}$ and for every $x \in \mathbf{R}$, the maps $y \mapsto \tilde{\pi} \circ \tilde{f}(x, y)$ and $y \mapsto \tilde{\pi} \circ \tilde{f}^{-1}(x, y)$ are both diffeomorphisms, the first one increasing and the second one decreasing. Iff is a positive twist map, $f^{-1}$ is a negative twist map. A twist map may be positive or negative.

Moreover, $f$ is exact symplectic if the 1 -form $f^{*}(r d \theta)-r d \theta$ is exact. 
Notations. $-\mathcal{M}_{\omega}^{+}$is the set of exact symplectic positive $\mathrm{C}^{1}$ twist maps of $\mathbf{A}, \mathcal{M}_{\omega}^{-}$is the set of exact symplectic negative $\mathrm{C}^{1}$ twist maps of $\mathbf{A}$ and $\mathcal{M}_{\omega}=\mathcal{M}_{\omega}^{+} \cup \mathcal{M}_{\omega}^{-}$is the set of exact symplectic $\mathbf{C}^{1}$ twist maps of $\mathbf{A}$.

It is obvious that if the graph $\mathrm{C}$ of a continuous map is invariant by $f \in \mathcal{M}_{\omega}$, then there exists no orbit for $f$ joining one of the connected component of $\mathbf{A} \backslash \mathrm{C}$ to the other one.

Birkhoff's theory states a kind of converse result (see [2, 6, 10, 12]):

Criterion (Birkhoff). - Let $\eta_{1}, \eta_{2}: \mathbf{T} \rightarrow \mathbf{R}$ be two continuous maps such that $\eta_{1}<\eta_{2}$. Let $f \in \mathcal{M}_{\omega}$. The three following properties are equivalent:

1. there exists no orbit under $f$ joining $\mathrm{S}_{-}\left(\eta_{1}\right)=\left\{(\theta, r) ; r<\eta_{1}(\theta)\right\}$ to $\mathrm{S}_{+}\left(\eta_{2}\right)=$ $\left\{(\theta, r) ; r>\eta_{2}(\theta)\right\}$;

2. there exists no orbit under $f$ joining $\mathrm{S}_{+}\left(\eta_{2}\right)$ to $\mathrm{S}_{-}\left(\eta_{1}\right)$;

3. there exists $\eta: \mathbf{T} \rightarrow \mathbf{R}$ continuous whose graph is invariant under $f$ such that: $\eta_{1} \leq \eta \leq \eta_{2}$.

Moreover, Birkhoff proved that if the graph of a continuous map $\eta$ is invariant under $f \in \mathcal{M}_{\omega}$, then $\eta$ is Lipschitz.

Having explained the link between the existence of invariant continuous (even Lipschitz) graphs of symplectic twist maps and the dynamic of such a twist map, we will now study the Lipschitz maps $\eta: \mathbf{T} \rightarrow \mathbf{R}$ whose graphs are invariant under an exact symplectic $\mathrm{C}^{\mathrm{l}}$ twist map.

We easily see that for every $\mathrm{C}^{1}$ map $\eta: \mathbf{T} \rightarrow \mathbf{R}$, there exists a $\mathrm{C}^{1}$ exact symplectic twist map $f:(\theta, r) \rightarrow(\theta+\varepsilon(r-\eta(\theta)), r-\eta(\theta)+\eta(\theta+\varepsilon(r-\eta(\theta))))$ (where $\varepsilon>0$ is small enough) which preserves the graph of $\eta$. But very few examples of Lipschitz but not $\mathrm{C}^{1}$ maps whose graph is invariant under an exact symplectic $\mathrm{C}^{1}$ twist map are known. The most classical example is the time $\mathrm{T}$ map $\Phi$ of the pendulum for $\mathrm{T}>0$ small enough (see [4]): any separatrix of the hyperbolic fixed point is a Lipschitz graph which is invariant under $\Phi$, and this graph is not differentiable at the fixed point. Of course, we can contruct similar examples:

Example. - Let $\alpha, \beta \in \mathbf{Z}^{*}$ be some integers and let $\mathcal{V}: \mathbf{T} \rightarrow \mathbf{R}$ be a small $\mathrm{C}^{2}$ function having a strict non degenerate global maximum at $0 \in \mathbf{T}$ (for example $\mathcal{V}(t)=$ $\varepsilon \cos t$ with $\varepsilon>0$ small enough). Let $\mathrm{V}: \mathbf{T}^{2} \rightarrow \mathbf{R}$ be defined by: $\mathrm{V}(x, t)=\mathcal{V}(\beta x-\alpha t)$ and let $\mathrm{H}: \mathbf{A} \times \mathbf{R} \rightarrow \mathbf{R}$ be the time dependent Hamiltonian function defined by: $\mathrm{H}(x, p ; t)=$ $\frac{1}{2} p^{2}+\mathrm{V}(x, t)$. The time 1 map of this Hamiltonian function is then a twist map, and if $(x, p)$ is a solution, if we define: $\mathrm{X}=\beta x-\alpha t$ and $\mathrm{P}=\beta p-\alpha$, then $(\mathrm{X}, \mathrm{P})$ is a solution for the (time-independent) Hamiltonian $\mathcal{H}(\mathrm{X}, \mathrm{P})=\frac{1}{2} \mathrm{P}^{2}+\beta^{2} \mathcal{V}(\mathrm{X})$. Therefore, there exists for the Hamiltonian flow of $\mathcal{H}$ (as for the pendulum) an invariant Lipschitz graph which is not differentiable at 0 ; then, the time 1 map of $\mathrm{H}$ leaves a Lipschitz graph invariant, and this Lipschitz graph is not differentiable at $\beta$ points of $\mathbf{T}$ (they correspond to a periodic orbit with period $\beta$ ); the rotation number of this graph is then $\frac{\alpha}{\beta}$. 
Questions.

1. Is it possible to construct less regular examples of invariant curves (which have at some points no left or right derivative)?

2. Does there exist an example of an invariant curve which is not $\mathrm{C}^{1}$ and has an irrational rotation number?

In this article, we don't answer these questions. We study the regularity of the curves invariant by exact symplectic $\mathrm{C}^{1}$ twist maps and prove that they are in general more regular than simply Lipschitz:

Theorem 1. - Letf $: \mathbf{A} \rightarrow \mathbf{A}$ be an exact symplectic positive $\mathrm{C}^{1}$ twist map and let $\boldsymbol{\gamma}: \mathbf{T} \rightarrow \mathbf{R}$ be a Lipschitz map whose graph is invariant by $f$. Then there exists a dense $\mathrm{G}_{\delta}$ subset $\mathrm{U}$ of $\mathbf{T}$ whose Lebesgue measure is 1 and such that every $t$ of $\mathrm{U}$ is a point of differentiability of $\gamma$ and a point of continuity of $\gamma^{\prime}$.

We endow the set of the Lipschitz maps $\gamma: \mathbf{T} \rightarrow \mathbf{R}$ with the metric $d_{\ell}$ defined by: $d_{\ell}\left(\gamma_{1}, \gamma_{2}\right)=d_{\infty}\left(\gamma_{1}, \gamma_{2}\right)+\operatorname{Lip}\left(\gamma_{1}-\gamma_{2}\right)$ where $\operatorname{Lip}(\gamma)$ is the Lipschitz constant of $\gamma$. This metric space $\left(\mathcal{L}, d_{\ell}\right)$ is then complete.

Corollary 2. - There exists a dense open subset $\mathcal{U}$ of $\left(\mathcal{L}, d_{\ell}\right)$ such that no $\gamma \in \mathcal{U}$ is invariant by an exact symplectic positive $\mathrm{C}^{1}$ twist map.

We obtain a stronger regularity if we can specify the dynamic of the restriction of the twist map to the curve:

Theorem 3. - Let $f: \mathbf{A} \rightarrow \mathbf{A}$ be an exact symplectic positive $\mathrm{C}^{1}$ twist map and let $\boldsymbol{\gamma}: \mathbf{T} \rightarrow \mathbf{R}$ be a Lipschitz map whose graph is invariant by $f$. Let $g$ be the restriction off to the graph of $\gamma$. We assume that there exist two sequences of integers $\left(n_{i}\right)_{i \in \mathbf{N}}$ and $\left(m_{i}\right)_{i \in \mathbf{N}}$ tending to $+\infty$ such that $\left(g^{m_{i}}\right)_{i \in \mathbf{N}}$ and $\left(g^{-n_{i}}\right)_{i \in \mathbf{N}}$ are equi-Lipschitz.

Then $\gamma$ is $\mathrm{C}^{\mathrm{l}}$.

Using a theorem of Michel Herman concerning the diffeomorphisms of the circle (see [9]), we deduce:

Corollary 4. - Let $f: \mathbf{A} \rightarrow \mathbf{A}$ be an exact symplectic positive $\mathrm{C}^{1}$ twist map and let $\gamma:$ $\mathbf{T} \rightarrow \mathbf{R}$ be a Lipschitz map whose graph is invariant by $f$. Let us assume that the restriction off to the graph of $\gamma$ is bi-Lipschitz conjugate to a rotation.

Then $\gamma$ is $\mathrm{G}^{1}$ and the restriction off to the graph of $\gamma$ is $\mathrm{C}^{1}$ conjugate to a rotation.

Now we are interested in studying the regularity of the exact symplectic $\mathrm{C}^{1}$ twist maps having many invariant curves: the $\mathrm{C}^{0}$ integrable ones. 
Definition. - Let $f: \mathbf{A} \rightarrow \mathbf{A}$ be an exact symplectic positive $\mathrm{C}^{1}$ twist map. Then $f$ is $\mathrm{C}^{0}$-integrable if $\mathbf{A}=\bigcup_{\gamma \in \Gamma} \mathrm{G}(\gamma)$ where:

1. $\Gamma$ is a subset of $\mathrm{C}^{0}(\mathbf{T}, \mathbf{R})$ and $\mathrm{G}(\gamma)$ is the graph of $\gamma$;

2. $\forall \gamma_{1}, \gamma_{2} \in \Gamma, \gamma_{1} \neq \gamma_{2} \Rightarrow \mathrm{G}\left(\gamma_{1}\right) \cap \mathrm{G}\left(\gamma_{2}\right)=\emptyset$;

3. $\forall \gamma \in \Gamma, f(\mathrm{G}(\gamma))=\mathrm{G}(\gamma)$.

Remark. - The general reference for this remark is [10].

A theorem of Birkhoff states that under the hypothesis of this definition, every $\gamma \in \mathrm{C}^{0}(\mathbf{T}, \mathbf{R})$ whose graph is invariant by $f$ is Lipschitz and that the set $\mathcal{I}(f)$ of those invariant graphs is closed for the $\mathrm{C}^{0}$-topology.

If we fix a lift $\tilde{f}$ of $f$, we can associate to every $\gamma \in \mathcal{I}(f)$ its rotation number $\rho(\gamma)$. Then, if $\gamma_{1}, \gamma_{2} \in \mathcal{I}(f)$, we have: $\mathrm{G}\left(\gamma_{1}\right) \cap \mathrm{G}\left(\gamma_{2}\right) \neq \emptyset \Rightarrow \rho\left(\gamma_{1}\right)=\rho\left(\gamma_{2}\right)$ and $\mathrm{G}\left(\gamma_{1}\right) \cap$ $\mathrm{G}\left(\gamma_{2}\right)=\emptyset \Rightarrow \rho\left(\gamma_{1}\right) \neq \rho\left(\gamma_{2}\right)$. We deduce that $\mathcal{I}(f)=\Gamma$ and therefore $\Gamma$ is closed for the $\mathrm{C}^{0}$ topology.

Theorem 5. - Let $f: \mathbf{A} \rightarrow \mathbf{A}$ be an exact symplectic positive $\mathrm{C}^{1}$ twist map which is $\mathbf{C}^{0}$ integrable. Let $\Gamma$ be the set of $\gamma \in \mathrm{C}^{0}(\mathbf{T}, \mathbf{R})$ whose graph is invariant under $f$. Then there exists a dense $\mathrm{G}_{\delta}$ subset $\mathcal{G}$ of $\left(\Gamma, d_{\infty}\right)$ such that: every $\gamma \in \mathcal{G}$ is $\mathrm{C}^{1}$. Moreover, in $\mathcal{G}$, the $\mathrm{G}^{0}$-topology is equal to the $\mathrm{C}^{1}$-topology.

There exists a common argument to the proof of all these results: the existence of two invariant (non continuous) subbundles along the invariant curves, the so-called "Green bundles".

The original Green bundles were introduced by L. W. Green in [8] for Riemannian geodesic flows; then P. Foulon extended this construction to Finsler metrics in [7] and G. Contreras and R. Iturriaga extended it in [5] to optical Hamiltonian flows; in [1], M. Bialy and R. S. Mackay give an analogous construction for the dynamics of sequence of symplectic twist maps of $\mathrm{T}^{*} \mathbf{T}^{d}$ without conjugate point. Let us cite also a very short survey [11] of R. Iturriaga on the various uses of these bundles (problems of rigidity, measure of hyperbolicity...).

The way we use the Green bundles in our article is different: the two Green bundles will bound the "derivative" below and above (this derivative is in fact the accumulation points of the slope between a given point and a variable one tending to the fixed one) of the invariant curve: therefore, if the two Green bundles are equal at one point, the curve has a derivative at this point.

\section{Construction of the Green bundles along an invariant curve}

Notations. $-\pi: \mathbf{T} \times \mathbf{R} \rightarrow \mathbf{T}$ is the projection.

If $x \in \mathbf{A}, \mathrm{V}(x)=\operatorname{ker} \mathrm{D} \pi(x) \subset \mathrm{T}_{x} \mathbf{A}$ is the vertical at $x$. 
If $x \in \mathbf{A}$ and $n \in \mathbf{N}, \mathrm{G}_{n}^{+}(x)=\mathrm{D} f^{n}\left(f^{-n}(x)\right) \mathrm{V}\left(f^{-n}(x)\right)$ and $\mathrm{G}_{n}^{-}(x)=$ $\mathrm{D} f^{-n}\left(f^{n}(x)\right) \mathrm{V}\left(f^{n}(x)\right)$ are two 1-dimensional linear subspaces (or lines) of $\mathrm{T}_{x} \mathbf{A}$.

Definition. - If we identify $\mathrm{T}_{x} \mathbf{A}$ with $\mathbf{R}^{2}$ by using the standard coordinates $(\theta, r) \in \mathbf{R}^{2}$, we may deal with the slope $s(\mathrm{~L})$ of any line $\mathrm{L}$ of $\mathrm{T}_{x} \mathbf{A}$ which is transverse to the vertical $\mathrm{V}(x)$ : it means that $\mathrm{L}=\{(t, s(\mathrm{~L}) t) ; t \in \mathbf{R}\}$.

If $x \in \mathbf{A}$ and if $\mathrm{L}_{1}, \mathrm{~L}_{2}$ are two lines of $\mathrm{T}_{x} \mathbf{A}$ which are transverse to the vertical $\mathrm{V}(x), \mathrm{L}_{2}$ is above (resp. strictly above) $\mathrm{L}_{1}$ if $s\left(\mathrm{~L}_{2}\right) \geq s\left(\mathrm{~L}_{1}\right)$ (resp. $\left.s\left(\mathrm{~L}_{2}\right)>s\left(\mathrm{~L}_{1}\right)\right)$. In this case, we write: $\mathrm{L}_{1} \preceq \mathrm{L}_{2}\left(\operatorname{resp} . \mathrm{L}_{1} \prec \mathrm{L}_{2}\right)$.

A sequence $\left(\mathrm{L}_{n}\right)_{n \in \mathbf{N}}$ of lines of $\mathrm{T}_{x} \mathbf{A}$ is non decreasing (resp. increasing) if for every $n \in \mathbf{N}$, $\mathrm{L}_{n}$ is transverse to the vertical and $\mathrm{L}_{n+1}$ is above (resp. strictly above) $\mathrm{L}_{n}$. We define the non increasing and decreasing sequences of lines of $\mathrm{T}_{x} \mathrm{M}$ in a similar way.

Remark. - A decreasing sequence of lines corresponds to a decreasing sequence of slopes.

Definition. - If $\mathrm{K}$ is a subset of $\mathbf{A}$ or of its universal covering $\mathbf{R} \times \mathbf{R}$, if $\mathbf{F}$ is a 1-dimensional subbundle of $\mathrm{T}_{\mathrm{K}} \mathbf{A}$ (resp. $\mathrm{T}_{\mathrm{K}} \mathbf{R}^{2}$ ) transverse to the vertical, we say that $\mathrm{F}$ is upper (resp. lower) semicontinuous if the map which maps $x \in \mathrm{K}$ onto the slope $s(\mathrm{~F}(x))$ of $\mathrm{F}(x)$ is upper (resp. lower) semicontinuous.

Notations. - If the graph of a continuous map $\gamma: \mathbf{T} \rightarrow \mathbf{R}$ is invariant by $f$, Birkhoff's theorem (see [10]) states that $\gamma$ is Lipschitz. Therefore, at every $t \in \mathbf{T}$, we can define:

$$
\gamma_{-}^{\prime}(t)=\liminf _{u \rightarrow t} \frac{\gamma(u)-\gamma(t)}{u-t} \quad \text { and } \quad \gamma_{+}^{\prime}(t)=\limsup _{u \rightarrow t} \frac{\gamma(u)-\gamma(t)}{u-t}
$$

which are two real numbers. We will use too:

$$
\gamma_{+, r}^{\prime}(t)=\limsup _{u \rightarrow t^{+}} \frac{\gamma(u)-\gamma(t)}{u-t} \text { and } \quad \gamma_{+, l}^{\prime}=\limsup _{u \rightarrow t^{-}} \frac{\gamma(u)-\gamma(t)}{u-t}
$$

and in a similar way $\gamma_{-, r}^{\prime}$ and $\gamma_{-, l}^{\prime}$.

(If $u$ is close enough to $t$, the difference $u-t$ is the unique real number of ]-0.5; 0.5[ which represents $u-t$.)

Proposition 6. - Let $f: \mathbf{T} \times \mathbf{R} \rightarrow \mathbf{T} \times \mathbf{R}$ be an exact symplectic positive $\mathrm{C}^{1}$ twist map and let $\gamma: \mathbf{T} \rightarrow \mathbf{R}$ be a Lipschitz map whose graph is invariant by $f$.

Then for every $t \in \mathbf{T}$ and every $n \in \mathbf{N}$, we have:

$$
\begin{aligned}
\mathrm{G}_{n}^{-}(t, \gamma(t)) & \prec \mathrm{G}_{n+1}^{-}(t, \gamma(t)) \prec \mathbf{R}\left(1, \gamma_{-}^{\prime}(t)\right) \preceq \mathbf{R}\left(1, \gamma_{+}^{\prime}(t)\right) \\
& \prec \mathrm{G}_{n+1}^{+}(t, \gamma(t)) \prec \mathrm{G}_{n}^{+}(t, \gamma(t)) .
\end{aligned}
$$


Notations. - If $\left(x_{1}, x_{2}\right) \in \mathbf{R}^{2}$, we will denote by $\mathcal{V}^{+}(x)$ the set: $\mathcal{V}^{+}(x)=\left\{\left(x_{1}, y\right) \in \mathbf{R}^{2}\right.$; $\left.y \geq x_{2}\right\}$.

Proof of Proposition 6. - Let $\tilde{f}: \mathbf{R} \times \mathbf{R} \rightarrow \mathbf{R} \times \mathbf{R}$ be a lift of $f$ and $\tilde{\gamma}: \mathbf{R} \rightarrow \mathbf{R}$ be defined by: $\tilde{\gamma}(\theta)=\gamma(\bar{\theta})$ where $\bar{\theta}$ is the projection of $\theta$ on $\mathbf{T}$. Then the graph $\mathrm{G}(\tilde{\gamma})$ is invariant by $\tilde{f}$ and every connected component of $\mathbf{R}^{2} \backslash \mathrm{G}(\tilde{\gamma})$ is invariant by $\tilde{f}$.

Let $x=(t, \tilde{\gamma}(t))$ be any point of $\mathrm{G}(\tilde{\gamma})$. We denote by $\mathrm{Q}(x)$ the connected component of $\mathbf{R}^{2} \backslash(\mathrm{G}(\tilde{\gamma}) \cup\{t\} \times \mathbf{R})$ which is above $\mathrm{G}(\tilde{\gamma})$ and in $] t,+\infty[\times \mathbf{R}$; moreover we denote by $\mathrm{R}(x)=\overline{\mathrm{Q}(x)}=\mathrm{Q}(x) \cup\left(\{(\tau, \tilde{\gamma}(\tau)) ; \tau \geq t\} \cup \mathcal{V}^{+}(x)\right)$ the closure of $\mathrm{Q}(x)$. The diffeomorphism $f$ being an exact symplectic positive $\mathrm{G}^{1}$ twist map, we have: $\forall x \in \mathrm{G}(\tilde{\gamma})$, $\tilde{f}(\mathrm{R}(x)) \subset \mathrm{R}(\tilde{f}(x))$. Therefore:

$$
\forall n \in \mathbf{N}^{*}, \forall x \in \mathrm{G}(\tilde{\gamma}), \quad \tilde{f}^{n}\left(\mathrm{R}\left(\tilde{f}^{-n}(x)\right)\right) \subset \tilde{f}^{n-1}\left(\mathrm{R}\left(\tilde{f}^{-(n-1)}(x)\right)\right) .
$$

We deduce that for every $n \in \mathbf{N}^{*}$ and every $x \in \mathrm{G}(\tilde{\gamma})$, the curve $\tilde{f}^{n}\left(\mathcal{V}^{+}\left(\tilde{f}^{-n}(x)\right)\right)$ is a subset of $\tilde{f}^{n-1}\left(\mathrm{R}\left(\tilde{f}^{-(n-1)}(x)\right)\right)$. Therefore, its tangent space at $x$, which is $\mathrm{G}_{n}^{+}(x)$ is under $\mathrm{G}_{n-1}^{+}(x)$ and above $\mathbf{R}\left(1, \gamma_{+, r}^{\prime}(t)\right)$. The fact that $\mathrm{G}_{n-1}^{+}(x)$ is strictly above $\mathrm{G}_{n}^{+}(x)$ follows from the fact that this subspaces have to be transverse because $\mathrm{V}\left(f^{-n-1}(x)\right)$ and $\mathrm{D} f\left(\mathrm{~V}\left(f^{-n}(x)\right)\right)$ are transverse ( $f$ being an exact symplectic positive $\mathrm{C}^{1}$ twist map). The fact that $\mathrm{G}_{n}^{+}(x)$ is strictly above $\mathbf{R}\left(1, \gamma_{+, r}^{\prime}(t)\right)$ comes then from the fact that the sequence $\left(\mathrm{G}_{n}^{+}(x)\right)$ is strictly decreasing.

The proof of the other inequalities is similar.

Remark. - In the last proof, we have noticed that if $x \in \mathrm{G}(\tilde{\gamma})$ the curve $\tilde{f}^{n}\left(\mathcal{V}^{+}\left(\tilde{f}^{-n}(x)\right)\right)$ is a subset of $\mathrm{R}(x)$ which is transverse to the vertical at $x$. Therefore, the first (or "horizontal") coordinate of $\mathrm{D} \tilde{f}^{n}\left(\tilde{f}^{-n}(x)\right)(0,1)$ is strictly positive.

Then $\left(\mathrm{G}_{n}^{+}(x)\right)$ is a strictly decreasing sequence of lines of $\mathrm{T}_{x} \mathbf{A}$ which is bounded below. Hence it tends to a limit $\mathrm{G}^{+}(x)$. In a similar way, the sequence $\left(\mathrm{G}_{n}^{-}(x)\right)$ tends to a limit, $\mathrm{G}^{-}(x)$.

Definition. - If $x \in \mathbf{A}$ belongs to a continuous graph invariant under $f \in \mathcal{M}_{\omega}^{+}$, the bundles $\mathrm{G}^{-}(x)$ and $\mathrm{G}^{+}(x)$ are called the Green bundles at $x$ associated to $f$.

Example. - Let us assume that $x \in \mathrm{G}(\gamma)$ is a periodic hyperbolic periodic point of $f$; then $\mathrm{G}^{+}(x)=\mathrm{E}^{u}(x)$ is the tangent space to the unstable manifold of $x$ and $\mathrm{G}^{-}(x)=$ $\mathrm{E}^{s}(x)$ is the tangent space to the stable manifold.

Proposition 7. - Let $\gamma: \mathbf{T} \rightarrow \mathbf{R}$ be a continuous map whose graph is invariant by an exact symplectic positive $\mathrm{C}^{1}$ twist map $f: \mathbf{A} \rightarrow \mathbf{A}$. Then the Green bundles, defined at every point of $\mathrm{G}(\gamma)$, are invariant by $\mathrm{D} f$ and for every $t \in \mathbf{T}$, we have: $\mathrm{G}^{-}(t, \gamma(t)) \preceq \mathbf{R}\left(1, \gamma_{-}^{\prime}(t)\right) \preceq$ 
$\mathbf{R}\left(1, \gamma_{+}^{\prime}(t)\right) \preceq \mathrm{G}^{+}(t, \gamma(t))$. Moreover, the map $t \rightarrow \mathrm{G}^{+}(t, \gamma(t))$ is upper semi-continuous and the map $t \rightarrow \mathrm{G}^{-}(t, \gamma(t))$ is lower semi-continuous. Therefore, the set:

$$
\mathcal{G}(\gamma)=\left\{t \in \mathbf{T} ; \mathrm{G}^{-}(t, \gamma(t))=\mathrm{G}^{+}(t, \gamma(t))\right\}
$$

is a $\mathrm{G}_{\delta}$ set and for every $t_{0} \in \mathcal{G}(\gamma), \gamma$ is differentiable, $\gamma^{\prime}$ is continuous at $t_{0}$ and $\mathbf{R}\left(1, \gamma^{\prime}\left(t_{0}\right)\right)=$ $\mathrm{G}^{+}\left(t_{0}, \gamma^{\prime}\left(t_{0}\right)\right)=\mathrm{G}^{-}\left(t_{0}, \gamma^{\prime}\left(t_{0}\right)\right)$. Moreover, $\mathrm{G}^{-}$and $\mathrm{G}^{+}$are continuous at $\left(t_{0}, \gamma\left(t_{0}\right)\right)$ too.

This proposition is a corollary of Proposition 6 and of usual properties of real functions (the fact that the (simple) limit of a decreasing sequence of continuous functions is upper semi-continuous).

Corollary 8. - Let $\gamma: \mathbf{T} \rightarrow \mathbf{R}$ be a continuous map whose graph is invariant by an exact symplectic positive $\mathrm{C}^{1}$ twist mapf $: \mathbf{A} \rightarrow \mathbf{A}$. We assume that:

$$
\forall t \in \mathbf{T}, \quad \mathrm{G}^{-}(t, \gamma(t))=\mathrm{G}^{+}(t, \gamma(t)) .
$$

Then $\gamma$ is $\mathrm{C}^{1}$.

Moreover, in this case, the sequences $\left(s\left(\mathrm{G}_{n}^{-}(t, \gamma(t))\right)\right)_{n \in \mathbf{N}}$ and $\left(s\left(\mathrm{G}_{n}^{+}(t, \gamma(t))\right)\right)_{n \in \mathbf{N}}$ converge uniformly to $\gamma^{\prime}(t)$.

Everything in this corollary is a consequence of Proposition 7; the fact that the convergence is uniform comes from Dini's theorem: if an increasing or decreasing sequence of real valued continuous functions defined on a compact set converges simply to a continuous function, then the convergence is uniform.

Example. - We may ask ourselves: if the graph of a $\mathrm{C}^{1}$ map $\gamma: \mathbf{T} \rightarrow \mathbf{R}$ is invariant under an exact symplectic positive $\mathrm{C}^{1}$ twist map $f$, do we necessarily have along the graph of $\gamma$ the equality $\mathrm{G}^{+}=\mathrm{G}^{-}$? We will show that the answer is no.

In fact, if $g: \mathbf{T} \rightarrow \mathbf{T}$ is any orientation preserving $\mathrm{C}^{1}$ diffeomorphism, we may "immerse" $g$ into an exact symplectic $\mathrm{C}^{1}$ twist map $f$. Let us explain this fact: let $\tilde{g}$ : $\mathbf{R} \rightarrow \mathbf{R}$ be any lift of $g$. We define $\tilde{f}: \mathbf{R}^{2} \rightarrow \mathbf{R}^{2}$ by:

$$
\tilde{f}(x, r)=\left(\tilde{g}(x)+r, \tilde{g}^{-1}(r+\tilde{g}(x))-x\right) .
$$

Then $\tilde{f}$ is a lift of an exact symplectic positive $\mathrm{C}^{1}$ twist map $f$ and we have: $\forall t \in \mathbf{T}$, $f(t, 0)=(g(t), 0)$.

If now we assume that $g$ has a hyperbolic periodic point $x_{0} \in \mathbf{T}$ (then $x_{0}$ is attracting or repulsing), $\left(x_{0}, 0\right)$ is a hyperbolic periodic point for $f$ and therefore $\mathrm{G}^{-}\left(x_{0}, 0\right) \neq$ $\mathrm{G}^{+}\left(x_{0}, 0\right)$.

Using Proposition 7, we will prove in the next section that if the graph of a continuous map $\gamma$ is invariant by an exact symplectic $\mathrm{C}^{1}$ twist map $f: \mathbf{A} \rightarrow \mathbf{A}$, then there exists a dense $\mathrm{G}_{\delta}$ subset $\mathrm{G}$ of $\mathbf{T}$ such that every $x \in \mathrm{G}$ is a point of differentiability of $\gamma$ and a point of continuity of $\gamma^{\prime}$. 


\section{Regularity of the invariant graphs}

We begin by giving a criterion to determine if a given vector is in one of the two Green bundles.

Proposition 9. - Let $f$ be an exact symplectic positive $\mathrm{C}^{1}$ twist map and let $\gamma: \mathbf{T} \rightarrow \mathbf{R}$ be a Lipschitz map whose graph $\mathrm{G}(\gamma)$ is invariant by $f$.

Let us assume that $x \in \mathrm{G}(\gamma)$ and that $v \in \mathrm{T}_{x} \mathbf{A}$ is such that the sequence $\left(\left|\mathrm{D}\left(\pi \circ f^{n}\right)(x) v\right|\right)_{n \in \mathbf{N}}$ doesn't tends to $+\infty$. Then $v \in \mathrm{G}^{-}(x)$. In a similar way, if the sequence $\left(\left|\mathrm{D}\left(\pi \circ f^{-n}\right)(x) v\right|\right)_{n \in \mathbf{N}}$ doesn't tends to $+\infty$, then $v \in \mathrm{G}^{+}(x)$.

Proof of Proposition 9. - We use the standard symplectic coordinates $(\theta, r)$ of $\mathbf{A}$ and we define for every $k \in \mathbf{Z}: x_{k}=f^{k}(x)$.

In these coordinates, the line $\mathrm{G}_{n}^{+}\left(x_{k}\right)$ is the graph of $\left(t \rightarrow s_{n}^{+}\left(x_{k}\right) t\right)\left(s_{n}^{+}\left(x_{k}\right)\right.$ is the slope of $\left.\mathrm{G}_{n}^{+}\left(x_{k}\right)\right)$ and the line $\mathrm{G}_{n}^{-}\left(x_{k}\right)$ is the graph of $\left(t \rightarrow s_{n}^{-}\left(x_{k}\right) t\right)$. Moreover, the matrix $\mathrm{M}_{n}\left(x_{k}\right)$ of $\mathrm{D} f^{n}\left(x_{k}\right)$ (for $n \geq 1$ ) is a symplectic matrix:

$$
\mathbf{M}_{n}\left(x_{k}\right)=\left(\begin{array}{ll}
a_{n}\left(x_{k}\right) & b_{n}\left(x_{k}\right) \\
c_{n}\left(x_{k}\right) & d_{n}\left(x_{k}\right)
\end{array}\right)
$$

with $\operatorname{det} \mathbf{M}_{n}\left(x_{k}\right)=1$. We have noticed just after the proof of Proposition 6 that the coordinate $\mathrm{D}\left(\pi \circ f^{n}\right)\left(x_{k}\right)(0,1)=b_{n}\left(x_{k}\right)$ is strictly positive. Using the definition of $\mathrm{G}_{n}^{+}\left(x_{k+n}\right)$, we obtain: $d_{n}\left(x_{k}\right)=s_{n}^{+}\left(x_{k+n}\right) b_{n}\left(x_{k}\right)$.

The matrix $\mathrm{M}_{n}\left(x_{k}\right)$ being symplectic, we have:

$$
\mathbf{M}_{n}\left(x_{k}\right)^{-1}=\left(\begin{array}{cc}
d_{n}\left(x_{k}\right) & -b_{n}\left(x_{k}\right) \\
-c_{n}\left(x_{k}\right) & a_{n}\left(x_{k}\right)
\end{array}\right)
$$

we deduce from the definition of $\mathrm{G}_{n}^{-}\left(x_{k}\right)$ that: $a_{n}\left(x_{k}\right)=-b_{n}\left(x_{k}\right) s_{n}^{-}\left(x_{k}\right)$. Finally, if we use the fact that $\operatorname{det} \mathrm{M}_{n}\left(x_{k}\right)=1$, we obtain:

$$
\mathbf{M}_{n}\left(x_{k}\right)=\left(\begin{array}{cc}
-b_{n}\left(x_{k}\right) s_{n}^{-}\left(x_{k}\right) & b_{n}\left(x_{k}\right) \\
-b_{n}\left(x_{k}\right)^{-1}-b_{n}\left(x_{k}\right) s_{n}^{-}\left(x_{k}\right) s_{n}^{+}\left(x_{k+n}\right) & s_{n}^{+}\left(x_{k+n}\right) b_{n}\left(x_{k}\right)
\end{array}\right) .
$$

Lemma 10. - There exists a constant $\mathrm{M}>0$ such that:

$$
\forall x \in \mathrm{G}(\gamma), \forall n \in \mathbf{N}^{*}, \quad \max \left\{\left|s_{n}^{+}(x)\right|,\left|s_{n}^{-}(x)\right|\right\} \leq \mathrm{M} .
$$

Proof of Lemma 10. - We deduce from Proposition 6 that: $\forall x \in \mathrm{G}(\gamma), \forall n \in \mathbf{N}^{*}$, $s_{1}^{-}(x) \leq s_{n}^{-}(x)<s_{n}^{+}(x) \leq s_{1}^{+}(x)$. Therefore, we only have to prove the inequalities of the lemma for $n=1$.

The real number $s_{1}^{-}(x)$, which is the slope of $\mathrm{D} f^{-1}(f(x)) \mathrm{V}(f(x))$, depends continuously on $x$, and is defined for every $x$ belonging to the compact subset $\mathrm{G}(\gamma)$. Hence it is uniformly bounded. The same argument proves that $s_{1}^{+}$is uniformly bounded on $\mathrm{G}(\gamma)$ and concludes the proof of Lemma 10. 
Lemma 11. - If $x \in \mathrm{G}(\gamma)$, we have: $\lim _{n \rightarrow \infty} b_{n}(x)=+\infty$.

Proof of Lemma 11. - We have: $\forall n, m \in \mathbf{N}^{*}, \forall i \in \mathbf{Z}, \mathbf{M}_{n+m}\left(x_{i}\right)=\mathbf{M}_{n}\left(x_{i+m}\right) \mathbf{M}_{m}\left(x_{i}\right)$. It implies: $\quad b_{n+m}\left(x_{i}\right)=b_{n}\left(x_{i+m}\right) b_{m}\left(x_{i}\right)\left(s_{m}^{+}\left(x_{i+m}\right)-s_{n}^{-}\left(x_{i+m}\right)\right)$ and: $\quad-b_{n+m}\left(x_{i}\right) s_{n+m}^{-}\left(x_{i}\right)=$ $b_{n}\left(x_{i+m}\right) s_{n}^{-}\left(x_{i+m}\right) b_{m}\left(x_{i}\right) s_{m}^{-}\left(x_{i}\right)-b_{n}\left(x_{i+m}\right)\left(b_{m}\left(x_{i}\right)\right)^{-1}-b_{n}\left(x_{i+m}\right) b_{m}\left(x_{i}\right) s_{m}^{+}\left(x_{i+m}\right) s_{m}^{-}\left(x_{i}\right)$.

Hence:

$$
\begin{aligned}
& -b_{n+m}\left(x_{i}\right) s_{n+m}^{-}\left(x_{i}\right) \\
& \quad=-b_{n+m}\left(x_{i}\right) s_{m}^{-}\left(x_{i}\right)-b_{n+m}\left(x_{i}\right)\left(b_{m}\left(x_{i}\right)\right)^{-2} \frac{1}{s_{m}^{+}\left(x_{i+m}\right)-s_{n}^{-}\left(x_{i+m}\right)} .
\end{aligned}
$$

Therefore:

$$
s_{n+m}^{-}\left(x_{i}\right)=s_{m}^{-}\left(x_{i}\right)+\left(b_{m}\left(x_{i}\right)\right)^{-2} \frac{1}{s_{m}^{+}\left(x_{i+m}\right)-s_{n}^{-}\left(x_{i+m}\right)} .
$$

In particular:

$$
s_{1+m}^{-}\left(x_{i}\right)=s_{m}^{-}\left(x_{i}\right)+\left(b_{m}\left(x_{i}\right)\right)^{-2} \frac{1}{s_{m}^{+}\left(x_{i+m}\right)-s_{1}^{-}\left(x_{i+m}\right)} .
$$

Using the constant $\mathrm{M}$ found via Lemma 10, we have:

$$
s_{1+m}^{-}\left(x_{i}\right) \geq s_{m}^{-}\left(x_{i}\right)+\frac{1}{2 \mathrm{M}\left(b_{m}\left(x_{i}\right)\right)^{2}} .
$$

Hence:

$$
s_{1+m}^{-}\left(x_{i}\right) \geq s_{1}^{-}\left(x_{i}\right)+\frac{1}{2 \mathrm{M}} \sum_{k=2}^{m} \frac{1}{\left(b_{k}\left(x_{i}\right)\right)^{2}} .
$$

The sequence $\left(s_{m}^{-}\left(x_{i}\right)\right)_{m \in \mathbf{N}^{*}}$ being convergent, we must have:

$$
\sum_{k=2}^{\infty} \frac{1}{\left(b_{k}\left(x_{i}\right)\right)^{2}}<\infty
$$

and thus: $\lim _{k \rightarrow \infty} b_{k}\left(x_{i}\right)=+\infty$.

Let us now prove Proposition 9. Let us assume that $v \in \mathrm{T}_{x} \mathbf{A}$ is such that the sequence $\left(\left|\mathrm{D}\left(\pi \circ f^{n}\right)(x) v\right|\right)_{n \in \mathbf{N}}$ doesn't tends to $+\infty$. Then there is a sequence $\left(k_{n}\right)_{n \in \mathbf{N}}$ of integers tending to $+\infty$ such that the sequence $\left(\left|\mathrm{D}\left(\pi \circ f^{k_{n}}\right)(x) v\right|\right)_{n \in \mathbf{N}}$ is bounded. If $v=\left(v_{1}, v_{2}\right)$, we have: $\mathrm{D}\left(\pi \circ f^{k_{n}}\right)(x) v=b_{k_{n}}(x)\left(v_{2}-s_{k_{n}}^{-}(x) v_{1}\right)$ and $\lim _{n \rightarrow \infty} b_{k_{n}}(x)=+\infty$. We deduce: $\lim _{n \rightarrow \infty}\left(v_{2}-s_{k_{n}}^{-}(x) v_{1}\right)=0$. The sequence $\left(s_{k_{n}}^{-}(x)\right)_{n \in \mathbf{N}}$ tends to the slope $s^{-}(x)$ of $\mathrm{G}^{-}(x)$, and then $v \in \mathrm{G}^{-}(x)$. 
Example. - Let us assume that the exact symplectic positive $\mathrm{C}^{1}$ twist map $f$ : $\mathbf{A} \rightarrow \mathbf{A}$ has a regular and proper integral, i.e. that there exists a $\mathrm{C}^{1}$ regular and proper function $\mathrm{H}: \mathbf{A} \rightarrow \mathbf{R}$ such that: $\forall x \in \mathbf{A}, \mathrm{H}(f(x))=\mathrm{H}(x)$. Then, for every $n \in \mathbf{Z}$, we have: $\mathrm{H}\left(f^{n}(x)\right)=\mathrm{H}(x)$ and then: $\mathrm{DH}\left(f^{n}(x)\right) \mathrm{D} f^{n}(x)=\mathrm{DH}(x)$ and $\|\operatorname{grad} \mathrm{H}(x)\|^{2}=$ $\mathrm{DH}(x) \operatorname{grad} \mathrm{H}(x)=\mathrm{DH}\left(f^{n}(x)\right) \mathrm{D} f^{n}(x) \operatorname{grad} \mathrm{H}(x)$ i.e. if we denote by (.|.) the usual scalar product and if $\|\cdot\|=\sqrt{(. \mid .)}$ :

$$
\left(\operatorname{grad} \mathrm{H}\left(f^{n}(x)\right) \mid \mathrm{D} f^{n}(x) \cdot \operatorname{grad} \mathrm{H}(x)\right)=\|\operatorname{grad} \mathrm{H}(x)\|^{2} .
$$

Let $\Gamma=\mathrm{H}^{-1}(c)$ be a curve invariant by $f$. If we use a good parametrization $\gamma: \mathbf{R} / \mathrm{TZ} \rightarrow$ $\mathbf{A}$ of $\Gamma$, the base $(\dot{\gamma}(t), \operatorname{grad} \mathrm{H}(\gamma(t)))$ is symplectic: the base is orthogonal, oriented, and $\|\dot{\gamma}(t)\|=\frac{1}{\|\operatorname{gradH}(\gamma(t))\|}$.

The image of this symplectic base by $\mathrm{D} f^{n}$ is symplectic too. This new symplectic base is: $\left(\mathrm{D} f^{n}(\gamma(t)) \dot{\gamma}(t), \mathrm{D} f^{n}(\gamma(t)) \operatorname{grad} \mathrm{H}(\gamma(t))\right)=\left(\lambda \dot{\gamma}\left(\tau_{n}\right), \mathrm{D} f^{n}(\gamma(t)) \operatorname{grad} \mathrm{H}(\gamma(t))\right)$ where $\gamma\left(\tau_{n}\right)=f^{n}(\gamma(t))$ and $\lambda \in \mathbf{R}$. Because this base is symplectic, we have: $1=$ $\lambda \omega\left(\dot{\gamma}\left(\tau_{n}\right), \mathrm{D} f^{n}(\gamma(t)) \operatorname{grad} \mathrm{H}(\gamma(t))\right)$, this last value being equal to:

$$
1=\frac{\lambda}{\left\|\operatorname{grad} \mathrm{H}\left(\gamma\left(\tau_{n}\right)\right)\right\|^{2}}\left(\operatorname{grad} \mathrm{H}\left(\gamma\left(\tau_{n}\right)\right) \mid \mathrm{D} f^{n}(\gamma(t)) \operatorname{grad} \mathrm{H}(\gamma(t))\right) .
$$

Using (*), we obtain: $\lambda=\frac{\| \operatorname{grad} \mathrm{H}\left(\gamma\left(\tau_{n}\right) \|^{2}\right.}{\|\operatorname{grad} \mathrm{H}(\gamma(t))\|^{2}}$; hence the sequence $\left(\mathrm{D} f^{n}(\gamma(t)) \dot{\gamma}(t)\right)_{n \in \mathbf{Z}}=$ $\left(\frac{\left\|\operatorname{grad} \mathrm{H}\left(\gamma\left(\tau_{n}\right)\right)\right\|^{2}}{\|\operatorname{grad} \mathrm{H}(\gamma(t))\|^{2}} \dot{\gamma}\left(\tau_{n}\right)\right)_{n \in \mathbf{N}}$ is bounded (and even uniformly bounded in $\left.t \in \mathbf{R}\right)$. Using Proposition 9, we deduce that:

$$
\forall x \in \Gamma, \quad \mathrm{G}^{-}(x)=\mathrm{G}^{+}(x) .
$$

Hence, if $f$ has a regular and proper integral, the two Green bundles are equal at every point.

Let us notice too that for every $c \in \mathbf{R}$, the restriction of $f$ to $\mathrm{H}^{-1}(c)$ is $\mathrm{C}^{1}$ conjugate to a rotation: indeed, with the notations introduced before, the sequence $\left(\left(\mathrm{D} f^{n} \circ \gamma\right) \dot{\gamma}\right)_{n \in \mathbf{Z}}$ is uniformly bounded. Let $g: \mathbf{R} / \mathrm{TZ} \rightarrow \mathbf{R} / \mathrm{TZ}$ be the unique $\mathrm{C}^{1}$-diffeomorphism such that: $\forall t \in \mathbf{R} / \mathrm{TZZ}, f(\gamma(t))=\gamma(g(t))$. Then: $\forall n \in \mathbf{Z}, f^{n}(\gamma(t))=\gamma\left(g^{n}(t)\right)$ and $\mathrm{D} g^{n}(t)=$ $\left(\mathrm{D} \gamma\left(g^{n}(t)\right)\right)^{-1}\left(\mathbf{D} f^{n} \circ \gamma(t)\right) \dot{\gamma}(t)$ is uniformly bounded in $n \in \mathbf{Z}$ and $t \in \mathbf{R} / \mathrm{TZZ}$. By a theorem of Michel Herman (Theorem 6.1.1 of [9]), it implies that $g$ and then $f_{\mid \Gamma}$ is $\mathbf{C}^{1}$ conjugate to a rotation.

Proposition 12. - Let $\gamma: \mathbf{T} \rightarrow \mathbf{R}$ be a Lipschitz map whose graph is invariant by an exact symplectic positive $\mathrm{C}^{1}$ twist map $f: \mathbf{A} \rightarrow \mathbf{A}$. Then for almost every $t \in \mathbf{T}$, the sequences $\left(\left|\mathrm{D}\left(\pi \circ f^{n}\right)(t, \gamma(t))\left(1, \gamma^{\prime}(t)\right)\right|\right)_{n \in \mathbf{N}}$ and $\left(\left|\mathrm{D}\left(\pi \circ f^{-n}\right)(t, \gamma(t))\left(1, \gamma^{\prime}(t)\right)\right|\right)_{n \in \mathbf{N}}$ don't tend to $+\infty$.

Proof of Proposition 12. - We define: $f(t, \gamma(t))=\left(f_{1}(t, \gamma(t)), f_{2}(t, \gamma(t))\right)=$ $(g(t), \gamma(g(t)))$. Then $g: \mathbf{T} \rightarrow \mathbf{T}$ is a (bi)-Lipschitz homeomorphism of $\mathbf{T}$ which is homotopic to $\mathrm{Id}_{\mathbf{T}}$. There exists a set $\mathrm{U} \subset \mathbf{T}$ whose Lebesgue measure is one and such 
that $\gamma$ is differentiable at every $x \in \mathrm{U}$. Then, for every $k \in \mathbf{Z}$ and every $x \in \mathrm{U}$, the map $g^{k}=\pi \circ f^{k}(., \gamma()$.$) is differentiable at x$; we have then: $\forall t \in \mathrm{U}, \forall k \in \mathbf{Z},\left(g^{k}\right)^{\prime}(t) \geq 0$. Let $\tilde{g}: \mathbf{R} \rightarrow \mathbf{R}$ be a lift of $g$. Then: $\forall k \in \mathbf{Z}, \forall t \in \mathbf{R}, \tilde{g}^{k}(t+1)=\tilde{g}^{k}(t)+1$. Therefore:

$$
\forall k \in \mathbf{Z}, \quad 1=\tilde{g}^{k}(t+1)-\tilde{g}^{k}(t)=\int_{\mathrm{U}}\left(g^{k}\right)^{\prime}(s) d s .
$$

Using Fatou's theorem, we obtain: $1 \geq \int_{\mathrm{U}} \liminf _{n \rightarrow \infty}\left(g^{n}\right)^{\prime}(s) d s$ and then for almost $t \in \mathrm{U}$, the sequence $\left(\left|\left(g^{n}\right)^{\prime}(t)\right|\right)_{n \in \mathbf{N}}$ doesn't tend to $+\infty$. As we have:

$$
\forall t \in \mathrm{U}, \forall n \in \mathbf{N}, \quad\left(g^{n}\right)^{\prime}(t)=\mathrm{D}\left(\pi \circ f^{n}\right)(t, \gamma(t))\left(1, \gamma^{\prime}(t)\right),
$$

we obtain the Proposition 12.

End of the Proof of Theorem 1. - We can now finish the proof of Theorem 1. Let $\gamma: \mathbf{T} \rightarrow \mathbf{R}$ be a Lipschitz map whose graph is invariant by an exact symplectic positive $\mathrm{C}^{1}$ twist map $f: \mathbf{A} \rightarrow \mathbf{A}$. By Proposition 12, there exists a subset $\mathrm{U}$ of $\mathbf{T}$ with Lebesgue measure 1 such that for every $t \in \mathrm{U}$, the sequences $\left(\left|\mathrm{D}\left(\pi \circ f^{n}\right)(t, \gamma(t))\left(1, \gamma^{\prime}(t)\right)\right|\right)_{n \in \mathbf{N}}$ and $\left(\left|\mathrm{D}\left(\pi \circ f^{-n}\right)(t, \gamma(t))\left(1, \gamma^{\prime}(t)\right)\right|\right)_{n \in \mathbf{N}}$ are well defined and doesn't tend to $+\infty$. By Proposition 9, for every $t \in \mathrm{U}$, we have: $\left(1, \gamma^{\prime}(t)\right) \in \mathrm{G}^{-}(t, \gamma(t)) \cap \mathrm{G}^{+}(t, \gamma(t))$. By Proposition 7, $\mathcal{G}(\gamma)$ is a dense $\mathrm{G}_{\delta}$ subset which contains $\mathrm{U}$ and thus the Lebesgue measure of $\mathcal{G}(\gamma)$ is 1 , and every point of $\mathcal{G}(\gamma)$ is a point of derivability of $\gamma$ and a point of continuity of $\gamma^{\prime}$.

Proof of Theorem 3. - Let $f: \mathbf{A} \rightarrow \mathbf{A}$ be an exact symplectic positive $\mathrm{C}^{1}$ twist map and let $\gamma: \mathbf{T} \rightarrow \mathbf{R}$ be a Lipschitz map whose graph is invariant by $f$. Let $g$ be the restriction of $f$ to the graph of $\gamma$. We assume that there exist two sequences of integers $\left(n_{i}\right)_{i \in \mathbf{N}}$ and $\left(m_{i}\right)_{i \in \mathbf{N}}$ tending to $+\infty$ such that $\left(g^{m_{i}}\right)_{i \in \mathbf{N}}$ and $\left(g^{-n_{i}}\right)_{i \in \mathbf{N}}$ are equi-Lipschitz with constant $\mathrm{K}$. We assume that $\mathrm{K}$ is a Lipschitz constant for $(t \rightarrow(t, \gamma(t))$ too. Then:

$$
\begin{aligned}
\forall t, u \in \mathbf{R}, \forall i \in \mathbf{N}, \quad & d\left(f^{m_{i}}(t, \gamma(t)), f^{m_{i}}(u, \gamma(u))\right) \\
& \leq \mathrm{K} d((t, \gamma(t)),(u, \gamma(u))) \leq \mathrm{K}^{2} d(u, t) ; \\
\forall t, u \in \mathbf{R}, \forall i \in \mathbf{N}, \quad & d\left(f^{-n_{i}}(t, \gamma(t)), f^{-n_{i}}(u, \gamma(u))\right) \\
& \leq \mathrm{K} d((t, \gamma(t)),(u, \gamma(u))) \leq \mathrm{K}^{2} d(u, t) .
\end{aligned}
$$

Let us now consider $t \in \mathbf{T}$; as $\gamma$ is Lipschitz, there exists a sequence $\left(t_{n}\right)_{n \in \mathbf{N}} \in \mathbf{T}^{\mathbf{N}}$ such that $\lim _{n \rightarrow \infty} t_{n}=t$ and the sequence $\left(\frac{\gamma(t)-\gamma\left(t_{n}\right)}{t-t_{n}}\right)_{n \in \mathbf{N}}$ tends to $\delta \in \mathbf{R}$. Then we have $(\tilde{f}$ is any lift of $f)$ :

$$
\forall i \in \mathbf{N}, \quad \mathrm{D} f^{m_{i}}(t, \gamma(t))(1, \delta)=\lim _{n \rightarrow \infty} \frac{1}{t-t_{n}}\left(\tilde{f}^{m_{i}}(t, \tilde{\gamma}(t))-\tilde{f}^{m_{i}}\left(t_{n}, \tilde{\gamma}\left(t_{n}\right)\right)\right)
$$

and

$$
\forall i \in \mathbf{N}, \quad \mathrm{D} f^{-n_{i}}(t, \gamma(t))(1, \delta)=\lim _{n \rightarrow \infty} \frac{1}{t-t_{n}}\left(\tilde{f}^{-n_{i}}(t, \tilde{\gamma}(t))-\tilde{f}^{-n_{i}}\left(t_{n}, \tilde{\gamma}\left(t_{n}\right)\right)\right)
$$


Hence: $\forall i \in \mathbf{N}, \max \left\{\left\|\mathrm{D} f^{m_{i}}(t, \gamma(t))(1, \delta)\right\|,\left\|\mathrm{D} f^{-n\}_{i}}(t, \gamma(t))(1, \delta)\right\| \leq \mathrm{K}^{2}\right.$. Therefore, by Proposition $9, \mathrm{G}^{-}(t, \gamma(t))=\mathrm{G}^{+}(t, \gamma(t))=\mathbf{R}\left(1, \gamma^{\prime}(t)\right)$. We deduce from Corollary 8 that $\gamma$ is $\mathrm{G}^{1}$.

Proof of Corollary 4. - Let $f: \mathbf{A} \rightarrow \mathbf{A}$ be an exact symplectic positive $\mathrm{C}^{1}$ twist map and let $\gamma: \mathbf{T} \rightarrow \mathbf{R}$ be a Lipschitz map whose graph is invariant by $f$. Let us assume that the restriction $g$ of $f$ to the graph of $\gamma$ is bi-Lipschitz conjugate to a rotation: there exists $\varphi: \mathbf{T} \rightarrow \mathrm{G}(\gamma)$ such that $\varphi$ and $\varphi^{-1}$ are Lipschitz and a rotation $\mathrm{R}: \mathbf{T} \rightarrow \mathbf{T}$ such that $\varphi \circ \mathrm{R} \circ \varphi^{-1}=g$. Then: $\forall n \in \mathbf{N}, g^{n}=\varphi \circ \mathrm{R}^{n} \circ \varphi^{-1}$; therefore, if $\mathrm{K}$ is a common Lipschitz constant of $\gamma, \varphi$ and $\varphi^{-1}$, as $\mathrm{R}$ is an isometry, we have:

$$
\begin{aligned}
\forall t, u \in \mathbf{R}, \quad d( & \left.f^{n}(t, \gamma(t)), f^{n}(u, \gamma(u))\right) \\
= & d\left(\varphi \circ \mathrm{R}^{n} \circ \varphi^{-1}(t, \gamma(t)), \varphi \circ \mathrm{R}^{n} \circ \varphi^{-1}(u, \gamma(u))\right) \\
\leq & \mathrm{K}^{3} d(t, u) \leq \mathrm{K}^{3} d((t, \gamma(t)),(u, \gamma(u))) .
\end{aligned}
$$

Hence $\left(g^{k}\right)_{k \in \mathbf{Z}}$ is equi-Lipschitz.

We deduce from Theorem 3 that $\gamma$ is $\mathrm{C}^{1}$.

Moreover, for every $k \in \mathbf{Z}$, we have: $\left\|\mathrm{D}\left(\pi \circ f^{k}\right)(t, \gamma(t))\left(1, \gamma^{\prime}(t)\right)\right\| \leq \mathrm{K}^{3}$; hence, $\pi \circ$ $f(., \gamma()$.$) is a \mathrm{C}^{1}$ diffeomorphism of $\mathbf{T}$ which satisfies the assumptions of Theorem 6.1.1 of [9]: therefore it is $\mathrm{C}^{1}$ conjugate to a rotation, and $f_{\mid \mathrm{G}(\gamma)}$ too.

\section{A generic property of Lipschitz functions}

We think that the results contained in this section should be known as folklore. by:

If $\theta \in \mathbf{R}$, its projection on $\mathbf{T}=\mathbf{R} / \mathbf{Z}$ is denoted by $\bar{\theta}$. We define on $\mathbf{T}$ a metric $d$

$$
\forall(\bar{\alpha}, \bar{\beta}) \in \mathbf{T}^{2}, \quad d(\bar{\alpha}, \bar{\beta})=\min _{\bar{\chi}=\bar{\alpha}, \bar{y}=\bar{\beta}}|x-y| .
$$

Moreover, $\lambda$ is the Lebesgue measure on $\mathbf{T}$.

Let $\mathcal{L}$ be the vector space of Lipschitz maps from $\mathbf{T}$ to $\mathbf{R}$. We define on $\mathcal{L}$ a map Lip by:

$$
\operatorname{Lip}(\gamma)=\sup _{\bar{x} \neq \bar{y}} \frac{|\gamma(\bar{x})-\gamma(\bar{y})|}{d(\bar{x}, \bar{y})} .
$$

We define on $\mathcal{L}$ a norm $\|\|=.\|.\|_{\infty}+\operatorname{Lip}$. Then $(\mathcal{L},\|\|$.$) is a Banach space.$

Lemma 13. - There exists a subset $\mathrm{A}$ of $\mathbf{T}$ such that, for every open and non-empty subset $\mathrm{U}$ of $\mathbf{T}, \lambda(\mathrm{U} \cap \mathrm{A})>0$ and $\lambda(\mathrm{U} \cap(\mathbf{T} \backslash \mathrm{A}))>0$. 
Proof of Lemma 13. - Let us introduce a notation: if $\mathrm{J}$ is a closed interval which is not a point and $\mu \in] 0,1\left[, \mathrm{C}_{\mu}(\mathrm{J})\right.$ is a Cantor subset of $\mathrm{J}$ such that: $\lambda(\mathrm{C}(\mathrm{J}))=\mu \lambda(\mathrm{J})$.

We define $\lambda_{0}=\frac{1}{3}$ and construct $\mathrm{C}_{0}=\mathrm{C}_{\lambda_{0}}([0,1])$. Then $\lambda\left(\mathrm{C}_{0}\right)=\frac{1}{3}$ and $[0,1] \backslash \mathrm{C}_{0}$ is the union of a countable family $\left(\mathrm{J}_{n}^{0}\right)_{n \in \mathbf{N}}$ of open intervals. Let us notice that the measure of each of these intervals is less than $\frac{1}{2}$.

We define $\lambda_{1}=\frac{1}{6}$ and for every $n \in \mathbf{N}$, we build $\mathrm{C}_{n}^{1}=\mathrm{C}_{\lambda_{1}}\left(\overline{\mathrm{J}}_{n}^{0}\right)$, a Cantor subset built in $\overline{\mathrm{J}}_{n}^{0}$. We define: $\mathrm{C}_{1}=\bigcup_{n \in \mathbf{N}} \mathrm{C}_{n}^{1}$. Then $\lambda\left(\mathrm{C}_{0} \cup \mathrm{C}_{1}\right)=\frac{1}{3}+\frac{1}{6} \cdot \frac{2}{3}=\frac{1}{3}+\frac{1}{3^{2}}$ and $[0,1] \backslash\left(\mathrm{C}_{0} \cup \mathrm{G}_{1}\right)$ is the union of a countable family $\left(\mathrm{J}_{n}^{1}\right)_{n \in \mathbf{N}}$ of open intervals. Let us notice that the measure of each of these intervals is less than $\frac{1}{4}$.

We repeat this construction: for every $n \in \mathbf{N}, \lambda_{n}\left(1-\frac{1}{2}\left(1-\frac{1}{3^{n}}\right)\right)=\frac{1}{3^{n+1}}$ is such that $\mathrm{C}_{j}^{n}=\mathrm{C}_{\lambda_{n}}\left(\bar{J}_{j}^{n}\right)$, we have: $\mathrm{C}_{n}=\bigcup_{j \in \mathbf{N}} \mathrm{C}_{j}^{n}, \lambda\left(\mathrm{C}_{0} \cup \cdots \cup \mathrm{C}_{n}\right)=\frac{1}{2}\left(1-\frac{1}{3^{n+1}}\right)$ and $[0,1] \backslash\left(\mathrm{C}_{0} \cup\right.$ $\left.\cdots \cup \mathrm{C}_{n}\right)$ is the union of a countable family $\left(\mathrm{J}_{j}^{n}\right)_{j \in \mathbf{N}}$ of open intervals. The measure of each of these intervals is less than $\frac{1}{2^{n}}$.

We define: $\mathrm{C}=\bigcup_{n \in \mathbf{N}} \stackrel{\mathrm{C}}{n}_{n}$.

Let now $\mathrm{J}=] a, b\left[\right.$ be an open interval in $[0,1]$. We choose $n \in \mathbf{N}$ such that $\frac{1}{2^{n}}<\frac{b-a}{4}$. As the measure of each $\mathrm{J}_{j}^{n}$ is less than $\frac{1}{2^{n}}$, the set $\mathrm{C}_{0} \cup \cdots \cup \mathrm{C}_{n}$ meets $] \frac{b+a}{2}-\frac{b-a}{4}, \frac{b+a}{2}+\frac{b-a}{4}[$; the set $\mathrm{C}_{0} \cup \cdots \cup \mathrm{C}_{n}$ being totally discontinuous, one open set $\mathrm{J}_{j}^{n}$ meets $] \frac{b+a}{2}-\frac{b-a}{4}, \frac{b+a}{2}+$ $\frac{b-a}{4}[$ and therefore is contained in $\mathrm{J}$. We know that:

$$
\begin{aligned}
\lambda\left(\mathrm{J}_{j}^{n} \backslash \mathrm{G}\right) & =\lambda\left(\mathrm{J}_{j}^{n} \backslash \bigcup_{k \geq n+1} \mathrm{G}_{k}\right)=\left(\prod_{i=n+1}^{\infty}\left(1-\lambda_{i}\right)\right) \lambda\left(\mathrm{J}_{j}^{n}\right) \\
& =\left(\prod_{i=n+1}^{\infty}\left(1-\frac{2}{3^{i+1}+3}\right)\right) \lambda\left(\mathrm{J}_{j}^{n}\right) .
\end{aligned}
$$

Therefore $\left.\lambda\left(\mathrm{J}_{j}^{n} \backslash \mathrm{C}\right) \in\right] 0, \lambda\left(\mathrm{J}_{j}^{n}\right)[$ and $\mathrm{J}$ meets $\mathrm{C}$ and $[0,1] \backslash \mathrm{C}$ in subsets which have a non zero measure.

Proposition 14. - There exists a dense and open subset $\mathcal{U}$ of $\mathcal{L}$ such that, for every $\gamma \in \mathcal{U}$, there exists a subset $\mathrm{U}_{\gamma} \subset \mathbf{T}$ such that $\lambda\left(\mathrm{U}_{\gamma}\right)>0$ and every $t \in \mathrm{U}_{\gamma}$ is a point of differentiability of $\gamma$ and a point of discontinuity of $\gamma^{\prime}$.

Notations. - If $\mathrm{A}$ is a subset of $\mathbf{R}$ (resp. $\mathbf{T}), \chi_{\mathrm{A}}$ is the characteristic function of A, i.e.: $\forall x \in \mathrm{A}, \chi_{\mathrm{A}}(x)=1$ and $\forall x \notin \mathrm{A}, \chi_{\mathrm{A}}(x)=0$.

Proof of Proposition 14. - We begin by exhibiting one example of $\eta: \mathbf{T} \rightarrow \mathbf{R}$ in $\mathcal{L}$ such that the derivative of $\eta$ has no point of continuity. Let $\mathrm{A} \subset \mathbf{T}$ be chosen as in Lemma 13: $\mathrm{A}$ is a set such that for every open and non empty subset $\mathrm{U}$ of $\mathbf{T}, \lambda(\mathrm{U} \cap \mathrm{A})>0$ and $\lambda(\mathrm{U} \cap(\mathbf{T} \backslash \mathrm{A}))>0$. Then the map: $\alpha: \mathbf{T} \rightarrow \mathbf{R}$ defined by: $\alpha(t)=\lambda(\mathbf{T} \backslash \mathrm{A}) \chi_{\mathrm{A}}(t)-$ $\lambda(\mathrm{A}) \chi_{\mathbf{T} \backslash A}(t)$ is such that: $\int_{\mathbf{T}} \alpha=0$. Hence, $\alpha$ has a primitive $\eta: \mathbf{T} \rightarrow \mathbf{R}$ defined by: $\forall \theta \in\left[0,1\left[, \eta(\bar{\theta})=\int_{[0, \bar{\theta}]} \alpha\right.\right.$. 
The function $\alpha$ being Lebesgue integrable, we have: for almost every $t \in \mathbf{T}, \eta$ is differentiable at $t$ and $\eta^{\prime}(t)=\alpha(t)$. Moreover, $\alpha$ being bounded, the map $\eta$ is Lipschitz. We denote by $\mathrm{D}$ the set of $t \in \mathbf{T}$ such that $\eta$ is differentiable at $t$ and $\eta^{\prime}(t)=\alpha(t)$. We have noticed that $\lambda(\mathrm{D})=1$. Moreover, if $\mathrm{J}$ is any open non empty interval of $\mathbf{T}$, by Lemma 13, $\mu(\mathrm{D} \cap \mathrm{J} \cap \mathrm{A})>0$ and $\mu(\mathrm{D} \cap \mathrm{J} \cap(\mathbf{T} \backslash \mathrm{A}))>0$. If $t \in \mathrm{D} \cap \mathrm{J} \cap \mathrm{A}, \eta$ is differentiable at $t$ and $\eta^{\prime}(t)=\alpha(t)=\lambda(\mathbf{T} \backslash \mathrm{A})=a>0$; if $t \in \mathrm{D} \cap \mathrm{J} \cap(\mathbf{T} \backslash \mathrm{A})$, then $\eta$ is differentiable at $t$ and $\eta^{\prime}(t)=\alpha(t)=-\lambda(\mathrm{A})=-b<0$. Then in every neighbourhood of any point of differentiability of $\eta$, there exists $t_{1}, t_{2}$ points of differentiability of $\eta$ such that $\eta^{\prime}\left(t_{1}\right)=a$ and $\eta^{\prime}\left(t_{2}\right)=-b$. It implies that $\eta^{\prime}$ is nowhere continuous.

Before going on with the proof, let us notice that the set of the point of continuity of any function is a $\mathrm{G}_{\delta}$ subset, and then measurable.

We consider a Lipschitz map $\gamma: \mathbf{T} \rightarrow \mathbf{R}$ and an open subset $\mathcal{U}$ of $\mathcal{L}$ which contains $\gamma$; there are two cases:

1. either for every $\gamma_{1} \in \mathcal{U}$, there exists $\mathrm{U} \subset \mathbf{T}$ such that $\lambda(\mathrm{U})>0$ and every $t \in \mathrm{U}$ is a point of differentiability of $\gamma_{1}$ and a point of discontinuity of $\gamma_{1}^{\prime}$;

2. or there exists $\gamma_{1} \in \mathcal{U}$ and $\mathrm{U} \subset \mathbf{T}$ such that $\lambda(\mathrm{U})=1$ and every $t \in \mathrm{U}$ is a point of differentiability of $\gamma_{1}$ and a point of continuity of $\gamma_{1}^{\prime}$.

In this last case, we will prove that there exists an open non empty subset $\mathcal{V} \subset \mathcal{U}$ such that: for every $\gamma_{2} \in \mathcal{V}$, there exists $\mathrm{U} \subset \mathbf{T}$ such that $\lambda(\mathrm{U})>0$ and every $t \in \mathrm{U}$ is a point of differentiability of $\gamma_{2}$ and a point of discontinuity of $\gamma_{2}^{\prime}$. If we succeed in proving that, the Proposition 14 is proved.

Let us now build $\mathcal{V}$. Let $\mathrm{D}\left(\gamma_{1}\right)$ be the set of the points of continuity of $\gamma_{1}^{\prime}$ and let $d\left(\gamma_{1}\right)$ be the set of the points of differentiability of $\gamma_{1}$ : we know that $\lambda\left(\mathrm{D}\left(\gamma_{1}\right)\right)=1$. Let $\varepsilon \in$ ]0, 1 [ be such that the ball centered at $\gamma_{1}+\varepsilon \eta$ with radius equal to $\varepsilon \frac{b+a}{8}$ is contained in $\mathcal{U}$ : this ball is then denoted by $\mathcal{V}$. As at the beginning of the proof, we denote by $\mathrm{D}$ the set of $t \in \mathbf{T}$ such that $\eta$ is differentiable at $t$ and $\eta^{\prime}(t)=\alpha(t)$. Let now $t_{0} \in \mathrm{D}\left(\gamma_{1}\right)$. As $t_{0}$ is a point of continuity of $\gamma_{1}^{\prime}$, there exists a neighbourhood $\mathrm{U}_{0}$ of $t_{0}$ in $\mathbf{T}$ such that: $\forall t \in \mathrm{U}_{0} \cap d\left(\gamma_{1}\right),\left|\gamma_{1}^{\prime}(t)-\gamma_{1}^{\prime}\left(t_{0}\right)\right|<\varepsilon \frac{b+a}{16}$. Let now $\gamma_{2} \in \mathcal{V}$ : then $\gamma_{2}=\gamma_{1}+\varepsilon \eta+u$ with $\|u\|_{\infty}+\operatorname{Lip}(u)<\varepsilon \frac{b+a}{8}$. Let $d(u)$ be the set of points of differentiability of $u$ and let $\mathrm{V}_{0}=\mathrm{U}_{0} \cap d\left(\gamma_{1}\right) \cap \mathrm{D} \cap d(u)$. Then $\lambda\left(\mathrm{V}_{0}\right)=\lambda\left(\mathrm{U}_{0}\right)>0$ and:

1. $u, \gamma_{1}$ and $\eta$ are differentiable at every $t \in \mathrm{V}_{0}$;

2. for every $t \in \mathrm{V}_{0}$, we have $\left|u^{\prime}(t)\right|<\varepsilon \frac{a+b}{8}$ because $\operatorname{Lip}(u)<\varepsilon \frac{a+b}{8}$;

3. for every $t, t^{\prime} \in \mathrm{V}_{0},\left|\gamma_{1}^{\prime}(t)-\gamma_{1}^{\prime}\left(t^{\prime}\right)\right|<\varepsilon \frac{b+a}{8}$ because $t, t^{\prime} \in \mathrm{U}_{0}$;

4. if $t \in \mathrm{V}_{0} \cap \mathrm{A}$, then $\eta^{\prime}(t)=a$ and if $t \in \mathrm{V}_{0} \cap(\mathbf{T} \backslash \mathrm{A})$, then $\eta^{\prime}(t)=-b$.

We deduce:

1. if $t \in \mathrm{V}_{0} \cap \mathrm{A}$, then:

$$
\gamma_{2}^{\prime}(t)=\gamma_{1}^{\prime}(t)+\varepsilon \eta^{\prime}(t)+u^{\prime}(t)>\varepsilon a+\gamma_{1}^{\prime}\left(t_{0}\right)-2 \varepsilon \frac{b+a}{8}
$$




$$
=\gamma_{1}^{\prime}\left(t_{0}\right)+\varepsilon\left(a-\frac{b+a}{4}\right)
$$

2. if $t \in \mathrm{V} \cap(\mathbf{T} \backslash \mathrm{A})$, then:

$$
\gamma_{2}^{\prime}(t)<-\varepsilon b+\gamma_{1}^{\prime}\left(t_{0}\right)+2 \varepsilon \frac{b+a}{8}=\gamma_{1}^{\prime}\left(t_{0}\right)+\varepsilon\left(-b+\frac{b+a}{4}\right) .
$$

We have: $-b+\frac{b+a}{4}<a-\frac{b+a}{4}$. Hence $\gamma_{2}^{\prime}$ is discontinuous at every point of $\mathrm{U}_{0}$. Finally, we have proved that for every $\gamma_{2} \in \mathcal{V}$, the Lebesque measure of the set of the points of discontinuity of $\gamma_{2}^{\prime}$ is non zero. This ends the proof.

Of course, Corollary 2 is a consequence of the last proposition and Theorem 1 .

\section{The $\mathrm{G}^{0}$ integrability}

In this section, we will prove Theorem 5 . We consider an exact symplectic $\mathrm{C}^{1}$ twist $\operatorname{map} f: \mathbf{A} \rightarrow \mathbf{A}$ which is $\mathrm{C}^{0}$ integrable and denote by $\Gamma$ the set of the $\mathrm{C}^{0}$-maps $\gamma: \mathbf{T} \rightarrow \mathbf{R}$ whose graph is invariant under $f$. Using the remark given in the introduction, we notice that: $\forall \gamma_{1}, \gamma_{2} \in \Gamma$, either $\gamma_{1}<\gamma_{2}$ or $\gamma_{1}>\gamma_{2}$. We endow $\Gamma$ with the order $\leq$ and the metric $d_{\infty}$ of the uniform convergence.

Let $\tilde{f}=\left(\tilde{f}_{1}, \tilde{f}_{2}\right): \mathbf{R}^{2} \rightarrow \mathbf{R}^{2}$ be a lift of $f$. For every $\gamma \in \mathrm{C}^{0}(\mathbf{T}, \mathbf{R}), \tilde{\gamma}$ is defined by: $\tilde{\gamma}(\theta)=\gamma(\bar{\theta})$. Then $\tilde{\Gamma}=\{\tilde{\gamma} ; \gamma \in \Gamma\}$ is also an ordered set, and the graph of every $\tilde{\gamma} \in \tilde{\Gamma}$ is invariant by $\tilde{f}$. For every $\gamma \in \Gamma$ we will denote by $\rho(\gamma)$ the rotation number of $\tilde{f}_{1}(., \tilde{\gamma}()):. \mathbf{R} \rightarrow \mathbf{R}$ (see [9] for the definition). Then it is proved in [10] (2.4.2) that $\rho: \Gamma \rightarrow \mathbf{R}$ is increasing; moreover, it is continuous.

Proposition 15. - Let $f: \mathbf{A} \rightarrow \mathbf{A}$ be an exact symplectic positive $\mathrm{C}^{1}$ twist map which is $\mathrm{C}^{0}$ integrable. If the graph of a continuous map $\gamma: \mathbf{T} \rightarrow \mathbf{R}$ is invariant by $f$ and if its rotation number $\rho(\gamma)=\frac{p}{q}$ is rational, then: $\forall \theta \in \mathbf{T}, f^{q}(\theta, \gamma(\theta))=(\theta, \gamma(\theta))$.

Proof of Proposition 15. - Let $\left(\gamma_{n}\right)_{n \in \mathbf{N}}$ be a decreasing sequence of elements of $\Gamma$ which tends to $\gamma$. Then: $\forall n \in \mathbf{N}, \rho\left(\gamma_{n}\right)>\frac{p}{q}=\rho(\gamma)$ and $\lim _{n \rightarrow \infty} \rho\left(\gamma_{n}\right)=\frac{p}{q}$. We may also choose $\gamma_{n}$ in such a way that: $\forall n \in \mathbf{N}, \rho\left(\gamma_{n}\right) \in \mathbf{R} \backslash \mathbf{Q}$.

Then, we have: $\forall k \in \mathbf{N}, \forall \theta \in \mathbf{R}, \tilde{f}_{1}^{q}\left(\theta, \tilde{\gamma}_{k}(\theta)\right) \neq \theta+p$. We deduce that for every $k \in \mathbf{N}$ : either $(*)_{1} \forall \theta \in \mathbf{R}, \tilde{f}_{1}^{q}\left(\theta, \tilde{\gamma}_{k}(\theta)\right)>\theta+p$ or $(*)_{2} \forall \theta \in \mathbf{R}, \tilde{f}_{1}^{q}\left(\theta, \tilde{\gamma}_{k}(\theta)\right)<\theta+p$. Using the fact that $\tilde{f}_{1}\left(., \tilde{\gamma}_{k}()\right)$ is increasing and the fact that $\tilde{f}_{1}\left(\theta+1, \tilde{\gamma}_{k}(\theta+1)\right)=\tilde{f}_{1}\left(\theta, \tilde{\gamma}_{k}(\theta)\right)+1$ : we deduce:

$(*)_{1}$ either: $\forall n \in \mathbf{N}^{*}, \forall \theta \in \mathbf{R}, \tilde{f}_{1}^{n q}\left(\theta, \tilde{\gamma}_{k}(\theta)\right)>\theta+n p ;$

$(*)_{2}$ or: $\forall n \in \mathbf{N}^{*}, \forall \theta \in \mathbf{R}, \tilde{f}_{1}^{n q}\left(\theta, \tilde{\gamma}_{k}(\theta)\right)<\theta+n p$;

and then: 
$(*)_{1}$ either: $\forall \theta \in \mathbf{R}, \rho\left(\gamma_{k}\right)=\lim _{n \rightarrow \infty} \frac{\tilde{f}_{1}^{n q}\left(\theta, \tilde{\gamma}_{k}(\theta)\right)-\theta}{n q} \geq \frac{p}{q}$;

$(*)_{2}$ or: $\forall \theta \in \mathbf{R}, \rho\left(\gamma_{k}\right)=\lim _{n \rightarrow \infty} \frac{\tilde{f}_{1}^{n q}\left(\theta, \tilde{\gamma}_{k}(\theta)\right)-\theta}{n q} \leq \frac{p}{q}$.

But we know that $\rho\left(\gamma_{k}\right)>\frac{p}{q}$; therefore, the case $(*)_{2}$ is impossible and we have: $\forall k \in \mathbf{N}$, $\forall \theta \in \mathbf{R}, \tilde{f}_{1}^{q}\left(\theta, \tilde{\gamma}_{k}(\theta)\right)>\theta+p$. We deduce that: $\forall \theta \in \mathbf{R}, \tilde{f}_{1}^{q}(\theta, \tilde{\gamma}(\theta)) \geq \theta+p$.

Using now a increasing sequence of elements of $\Gamma$ tending to $\gamma$, we obtain, similarly: $\forall \theta \in \mathbf{R}, \tilde{f}_{1}^{q}(\theta, \tilde{\gamma}(\theta)) \leq \theta+p$.

Proof of Theorem 5. - Let $\mathcal{C}=\left\{\gamma \in \Gamma ; \gamma \in \mathrm{C}^{1}(\mathbf{T}, \mathbf{R})\right.$ and $\forall \theta \in \mathbf{T}, \mathrm{G}^{-}(\theta, \gamma(\theta))=$ $\left.\mathrm{G}^{+}(\theta, \gamma(\theta))\right\}$. By Corollary 8, we know that the condition $\gamma \in \mathrm{C}^{1}(\mathbf{T}, \mathbf{R})$ is redundant.

Lemma 16. - If $\gamma \in \Gamma$ is such that $\rho(\gamma)=\frac{p}{q} \in \mathbf{Q}$ then $\gamma \in \mathcal{C}$.

Proof of Lemma 16. - Let $\gamma \in \Gamma$ be such that $\rho(\gamma) \in \mathbf{Q}$. We deduce from Proposition 15 that every $(\theta, \gamma(\theta))$ is $q$-periodic for $f$.

Hence if $g$ is the restriction of $f$ to the graph $\mathrm{G}(\gamma)$ of $\gamma$, the family $\left(g^{n q}\right)_{n \in \mathbf{Z}}=$ $\left(\mathrm{I} d_{\mathrm{G}(\gamma)}\right)_{n \in \mathbf{Z}}$ is equi-Lipschiz. We deduce from Theorem 3 and from its proof that $\gamma \in \mathcal{C}$.

We define: $\Gamma_{0}=\{\gamma \in \Gamma ; \rho(\gamma) \in \mathbf{Q}\}$; then $\Gamma_{0}$ is dense in $\Gamma$; Lemma 16 implies that: $\Gamma_{0} \subset \mathcal{C}$. Hence $\mathcal{C}$ is dense in $\Gamma$.

Let us now prove:

Lemma 17. - The map: $\mathbf{F}: \mathbf{T} \times \Gamma \rightarrow \mathbf{A}$ defined by: $\mathrm{F}(\theta, \gamma)=(\theta, \gamma(\theta))$ is a homeomorphism.

Proof of Lemma 17. - This map is continuous, one-to-one and onto. Moreover, a result due to Birkhoff states that for every compact set $\mathrm{K}$ of $\mathbf{A}$, the set $\{\gamma \in \Gamma ; \mathrm{G}(\gamma) \cap \mathrm{K} \neq$ $\emptyset\}$ is compact. Therefore the map $\mathrm{F}$ is proper; hence, $\mathrm{F}$ is a homeomorphism.

Lemma 18. - The set $\mathcal{G}=\left\{x \in \mathbf{A} ; \mathrm{G}^{+}(x)=\mathrm{G}^{-}(x)\right\}$ is a $\mathrm{G}_{\delta}$ subset of $\mathbf{A}$.

The proof is the same as in Proposition $7: \mathrm{G}^{+}$is upper semi-continuous and $\mathrm{G}^{-}$is lower semi-continuous.

The map $\mathrm{F}$ being a homeomorphism, we deduce from Lemma 18 that $\mathcal{G}=$ $\left\{(\theta, \gamma) \in \mathbf{T} \times \Gamma ; \mathrm{G}^{-}(\theta, \gamma(\theta))=\mathrm{G}^{+}(\theta, \gamma(\theta))\right\}$ is a $\mathrm{G}_{\delta}$ subset of $\mathbf{T} \times \Gamma$. Moreover, it contains $\mathbf{T} \times \mathcal{C}$ which is dense in $\mathbf{T} \times \Gamma$. Hence $\mathcal{G}$ is a dense $\mathrm{G}_{\delta}$ subset of $\mathbf{T} \times \Gamma$. Therefore there exists a sequence $\left(\mathrm{U}_{n}\right)_{n \in \mathbf{N}}$ of open subsets of $\mathbf{T} \times \Gamma$ such that $\mathcal{G}=\bigcap_{n \in \mathbf{N}} \mathrm{U}_{n}$. If $n \in \mathbf{N}$, then $\mathbf{T} \times \mathcal{C} \subset \mathrm{U}_{n}$. As every set $\mathbf{T} \times\{\gamma\}$ is compact, the set $\mathrm{V}_{n}=\left\{\gamma \in \Gamma ; \mathbf{T} \times\{\gamma\} \subset \mathrm{U}_{n}\right\}$ contains an open subset $\mathrm{W}_{n}$ of $\Gamma$ which contains $\mathcal{C}$; then $\mathbf{T} \times \mathrm{W}_{n}$ is a dense and open subset of $\mathbf{T} \times \Gamma$ such that: $\mathbf{T} \times \mathcal{C} \subset \mathbf{T} \times \mathrm{W}_{n} \subset \mathrm{U}_{n}$. We deduce that $\mathrm{G}=\bigcap_{n \in \mathbf{N}} \mathrm{W}_{n}$ is a $\mathrm{G}_{\delta}$ of $\Gamma$ such that: $\mathbf{T} \times \mathcal{C} \subset \mathbf{T} \times \mathrm{G} \subset \mathcal{G}$. Hence $\mathrm{G}$ is a dense $\mathrm{G}_{\delta}$ subset of $\Gamma$ such that: $\forall \gamma \in \mathrm{G}, \mathbf{T} \times\{\gamma\}$ is a subset in $\mathcal{G}$; using Corollary 8 , we deduce that every $\gamma \in \mathrm{G}$ is $\mathrm{C}^{1}$. 


\section{Acknowledgement}

I am grateful to J.-C. Yoccoz who pointed to me the beautifull results of M. Herman and to S. Crovisier and A. Fathi for their comments on the original manuscript.

\section{REFERENCES}

1. M. L. Bialy and R. S. MacKay, Symplectic twist maps without conjugate points, Isr. F. Math., 141 (2004), $235-247$.

2. G. D. Birkhoff, Surface transformations and their dynamical application, Acta Math., 43 (1920), 1-119.

3. A. Chenciner, La dynamique au voisinage d'un point fixe elliptique conservatif: de Poincaré et Birkhoff à Aubry et Mather., Astérisque, 1983/84(121-122) (1985), 147-170 (in French). [The dynamics at the neighborhood of a conservative elliptic fixed point: from Poincare and Birkhoff to Aubry and Mather] Seminar Bourbaki.

4. A. Chenciner, Systèmes dynamiques différentiables. Article à l'Encyclopedia Universalis.

5. G. Contreras and R. Iturriaga, Convex Hamiltonians without conjugate points, Ergod. Theory Dyn. Syst., 19(4) (1999), 901-952.

6. А. FAтнi, Une interprétation plus topologique de la démonstration du théorème de Birkhoff, appendice au ch.1 de [9], pp. 39-46.

7. P. Foulon, Estimation de l'entropie des systèmes lagrangiens sans points conjugués, Ann. Inst. Henri Poincaré Phys. Théor., $\mathbf{5 7}(2)(1992), 117-146$.

8. L. W. Green, A theorem of E. Hopf, Mich. Math. F., 5 (1958), 31-34.

9. M. Herman, Sur la conjugaison différentiable des difféomorphismes du cercle à des rotations, Inst. Hautes Étud. Sci. Publ. Math., 49 (1979), 5-233.

10. M. Herman, Sur les courbes invariantes par les difféomorphismes de l'anneau, Asterisque, 1 (1983), 103-104.

11. R. Iturriaga, A geometric proof of the existence of the Green bundles, Proc. Amer. Math. Soc., 130(8) (2002), 23112312.

12. P. Le Calvez, Etude topologique des applications déviant la verticale, Ens. Mat., Soc. Bras. Mat., 2 (1990).

M.-C. A.

Université d'Avignon et des Pays de Vaucluse

Laboratoire d'Analyse Non Linéaire

et Géométrie (EA 2151)

84018 Avignon, France

Marie-Claude.Arnaud@univ-avignon.fr

Manuscrit reçu le 5 avril 2007

publié en ligne le 19 février 2009. 